\title{
The crucial role of Campylobacter jejuni genes in anti-ganglioside antibody induction in Guillain-Barré syndrome
}

\author{
Peggy C.R. Godschalk, ${ }^{1}$ Astrid P. Heikema, ${ }^{1}$ Michel Gilbert, ${ }^{2}$ Tomoko Komagamine, ${ }^{3}$ C. Wim Ang, ${ }^{1}$ \\ Jobine Glerum, ${ }^{1}$ Denis Brochu, ${ }^{2}$ Jianjun Li, ${ }^{2}$ Nobuhiro Yuki, ${ }^{3}$ Bart C. Jacobs, ${ }^{4,5}$ \\ Alex van Belkum, ${ }^{1}$ and Hubert P. Endtz ${ }^{1}$ \\ 1Department of Medical Microbiology and Infectious Diseases, Erasmus MC, University Medical Center, Rotterdam, The Netherlands. \\ Institute for Biological Sciences, National Research Council of Canada, Ottawa, Ontario, Canada. ${ }^{3}$ Department of Neurology, \\ Dokkyo University School of Medicine, Shimotsuga, Tochigi, Japan. ${ }^{4}$ Department of Neurology and ${ }^{5}$ Department of Immunology, \\ Erasmus MC, University Medical Center, Rotterdam, The Netherlands.
}

\begin{abstract}
Molecular mimicry of Campylobacter jejuni lipo-oligosaccharides (LOS) with gangliosides in nervous tissue is considered to induce cross-reactive antibodies that lead to Guillain-Barré syndrome (GBS), an acute polyneuropathy. To determine whether specific bacterial genes are crucial for the biosynthesis of ganglioside-like structures and the induction of anti-ganglioside antibodies, we characterized the C. jejuni LOS biosynthesis gene locus in GBS-associated and control strains. We demonstrated that specific types of the LOS biosynthesis gene locus are associated with GBS and with the expression of ganglioside-mimicking structures. Campylobacter knockout mutants of 2 potential GBS marker genes, both involved in LOS sialylation, expressed truncated LOS structures without sialic acid, showed reduced reactivity with GBS patient serum, and failed to induce an anti-ganglioside antibody response in mice. We demonstrate, for the first time, to our knowledge, that specific bacterial genes are crucial for the induction of anti-ganglioside antibodies.
\end{abstract}

\section{Introduction}

Autoimmune diseases are often preceded by an infectious illness. Molecular mimicry between microbial antigens and structures in host tissue has been implicated as a mechanism for triggering a crossreactive immune response after an infection (1). There is strong but indirect evidence for the pathogenic role of molecular mimicry in Guillain-Barré syndrome (GBS), an acute peripheral polyneuropathy and the most frequent cause of acute neuromuscular paralysis (2). Therefore, GBS is an excellent model disease to study both microbial and host factors involved in molecular mimicry.

The most frequently identified triggering agent of GBS is Campylobacter jejuni, a spiral-shaped Gram-negative bacterium $(3,4)$. C. jejuni is the leading causative agent of bacterial gastroenteritis worldwide, and it has recently also been associated with neoplastic disease of the gut (5). Acute-phase sera of GBS patients contain high titers of antibodies directed against gangliosides, membrane glycolipids that are highly enriched in nervous tissue (6). Biochemical and serological studies have identified various gangliosidemimicking structures in the lipo-oligosaccharides (LOS) of the Campylobacter cell wall (7), and cross-reactive antibodies between Campylobacter LOS and gangliosides have been demonstrated in serum from GBS patients (6). Ganglioside-mimicking structures are found more frequently in neuropathy-associated C. jejuni strains than in strains isolated from patients with diarrhea (8). An important feature in ganglioside mimicry is the presence of sialic acid ( $N$-acetylneuraminic acid) in both LOS and gangliosides (9).

Nonstandard abbreviations used: $\mathrm{Cm}^{\mathrm{r}}$, chloramphenicol resistance; cst-II, campylobacter sialic acid transferase-II; GBS, Guillain-Barré syndrome; LOS, lipo-oligosaccharides; MFS, Miller Fisher syndrome; orf, open reading frame. Conflict of interest: The authors have declared that no conflict of interest exists.

Citation for this article: J. Clin. Invest. 114:1659-1665 (2004).

doi:10.1172/JCI200415707.
Recently, we described a collection of geographically clustered but genetically heterogeneous C. jejuni strains isolated from Dutch patients with GBS or its variant, Miller Fisher syndrome (MFS) (10, 11). Characterization of the isolates by phenotypic and molecular methods showed that no clustering of GBS/MFS-associated strains occurred when these were compared with control strains $(10,12)$.

The availability of a database with detailed serological and clinical data of the Dutch GBS/MFS patients provides a unique opportunity for a systematic search for bacterial GBS/MFS-associated virulence factors and correlations with specific immune responses and clinical presentation. We recently reported the association between the presence of the campylobacter sialic acid transferase-II (cst-II) gene and the expression of a GQ1b-like structure in the bacterial LOS (13). Based on these findings, we hypothesized that the presence of certain C. jejuni genes involved in LOS biosynthesis may be crucial for the induction of the anti-ganglioside immune response that leads to GBS. Therefore, we analyzed the LOS biosynthesis gene locus of GBS/MFS-associated C. jejuni strains. We found that specific types of the LOS biosynthesis locus are associated with GBS, and this finding led to the identification of potential GBS marker genes in C. jejuni. Functional analysis of Campylobacter gene knockout mutants, including mouse immunization experiments, demonstrated that these genes are crucial for the induction of anti-ganglioside antibodies.

\section{Results}

Specific classes of the LOS biosynthesis gene locus are associated with neuropathy and ganglioside mimicry. In C. jejuni, the genes involved in LOS biosynthesis are clustered in the LOS biosynthesis gene locus (LOS locus) (14). Variation in gene content of the LOS locus is an important mechanism for variation in LOS structure between different C. jejuni strains. Previously, we had described 3 different gene compositions or "classes" of the LOS locus in C. jejuni (15). 


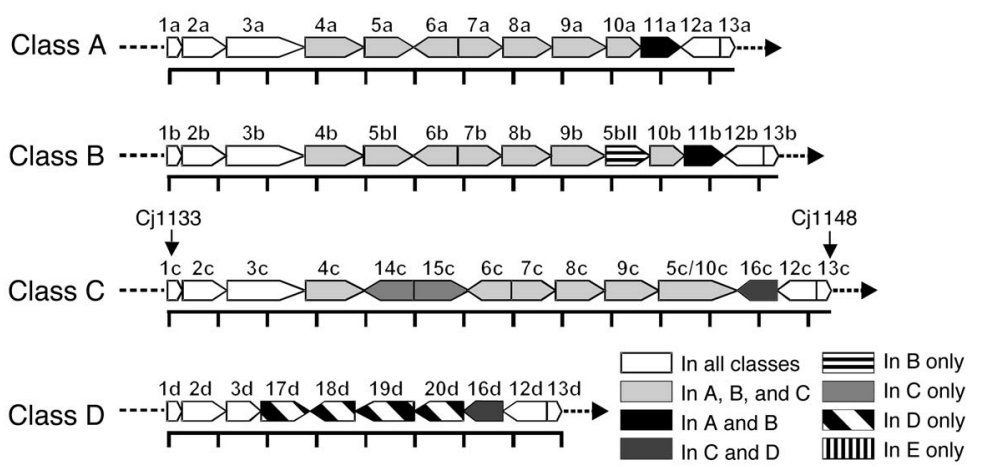
Class E -..-.

\section{Figure 1}

Genetic organization of the 5 different classes of the $C$. jejuni LOS biosynthesis locus. The distance between the scale marks is $1 \mathrm{~kb}$. The direction of the arrows indicates the direction of transcription. Corresponding homologous genes have the same number with a letter for the LOS locus class added. For orf1c and orf $13 \mathrm{c}$ the corresponding $\mathrm{Cj}$ gene numbers of the "genome" strain NCTC 11168 are given. The 5 LOS classes are based on DNA sequences of the following strains (GenBank accession number): class A: OH4384 (AF130984), OH4382 (AF167345), HS:4 (AF215659), HS:10 (AF400048), HS:19 (AF167344), HS:41 (AY044868); class B: HS:23 (AF401529), HS:36 (AF401528); class C: NCTC 11168 (AL139077), HS:1 (AY044156), HS:2 (AF400047); class D: LIO87 (AF400669); class E: 81116 (AF343914 and AJ131360). The proposed functions for the orfs are described in Supplemental Table 1.

Since then, the DNA sequences of several additional C. jejuni LOS loci were deposited in GenBank (http://www.ncbi.nlm.nih.gov/ Genbank/), and there are now 5 distinct classes (Figure 1).

To study whether certain classes are more prevalent among neuropathy-associated C. jejuni strains, we determined the class of LOS locus (classes A-E) in a collection of 21 neuropathy-associated and 21 control C. jejuni strains isolated from patients with uncomplicated enteritis. All the strains used in this study were positive for 1 of the 5 identified LOS locus classes. In addition, we analyzed the individual class A/B and class $\mathrm{C}$ genes by PCR RFLP and hybridization analysis and found that the LOS gene content in the strains was in agreement with their class of LOS locus (data not shown). The class A LOS locus was overrepresented in the GBS-associated strains compared with the control strains $(53 \%$ vs. $14 \%, P=0.02$; Table 1). In contrast, all 4 MFS-associated strains contained a class B locus, which was detected in only $33 \%$ of control strains $(P=0.03$; Table 1$)$ and $18 \%$ of GBS-associated strains $(P<0.01)$.

Studying the expression of ganglioside-like structures in relation to the class of LOS locus, we found that GM1-like structures were associated with a class A locus $(P<0.01)$, whereas GQ1b-like structures were predominantly expressed by strains with a class $B$ locus $(P<0.01$; Table 2$)$. Although the class A locus was associated with both GBS and the presence of a GM1-like structure, a GM1like structure was not detected more frequently in GBS-associated strains (13 of 17 GBS strains vs. 12 of 21 control strains, $P=0.3$ ). In 8 of 11 strains with a class D or E locus, ganglioside-like structures were not detected, which is in accordance with the absence of genes involved in the biosynthesis or transfer of sialic acid in these classes (Table 2; Figure 1). These results indicate that genes that are unique to the class $\mathrm{A}$ and $\mathrm{B}$ loci and genes that are involved in sialic acid biosynthesis or transfer may be crucial for the induction of neuropathogenic cross-reactive antibodies and may thus be considered as GBS marker genes.

Mutagenesis of putative GBS marker genes. To study whether potential GBS marker genes are indeed crucial for the induction of potentially neuropathogenic cross-reactive antibodies, we constructed Campylobacter knockout mutants of 3 potential GBS marker genes. Open reading frame 11 (orf11) is unique to the class A and B loci (Figure 1). Its function is unknown, but sequence homology with an enzyme of Rhodobacter capsulatus suggests that orf11 encodes an acetyltransferase (16). The cst-II gene (orf7) encodes an enzyme (Cst-II) that is involved in the transfer of sialic acid to the LOS backbone. Orf10 encodes a CMP-sialic acid synthetase and is involved in the biosynthesis of the sialic acid donor used by Cst-II.

Cst-II and orf11 single mutants and an orf10/orf11 double mutant were produced in 2 class A GM1-expressing GBS-associated C. jejuni strains (GB2 and GB11) by insertion of a chloramphenicol resistance $\left(\mathrm{Cm}^{\mathrm{r}}\right)$ cassette (Figure 2). The cassette was inserted with the same transcriptional polarity as that of the mutated genes. For all mutants, possible double crossover events were verified by PCR. Southern blot analysis confirmed single integration of the $\mathrm{Cm}^{\mathrm{r}}$ cassette into the correct position on the chromosome (data not shown).

LOS structure analysis of WTs and mutants. To determine the effect of the gene inactivations on the LOS structure, we performed mass spectrometry analysis and identified a mixture of GM1- and GD1a-like structures for both GB2 and GB11 WT LOS (Figure 3). The sets of triply charged ions corresponding to GM1- and GD1a-like structures were of similar intensities (data not shown), which suggests that these 2 structures are present in approximately equimolar amounts, assuming that the ionization efficiency is proportional to the amount of a species in the sample. Both the cst-II and the orf10/ orf11 mutants of GB2 and GB11 expressed a mixture of 3 nonsialylated structures. The main fraction consisted of asialo-GM3, but asialo-GM2 and asialo-GM1 structures were also present (Figure 3). No structural differences were observed between the WTs and the orf11 mutants. This indicates that the truncated LOS structures as observed in the orf10/orf11 mutants were caused by the inactivation of orf10 and not orf11. Detailed results of the mass spectrometry analysis can be found in Supplemental Table 2 (supplemental material available at http://www.jci.org/cgi/content/full/114/11/1659/DC1).

\section{Table 1}

LOS biosynthesis loci in $C$. jejuni strains from patients with GBS, MFS, and uncomplicated enteritis

\begin{tabular}{lcccc}
\hline \multicolumn{5}{c}{ No. of strains associated with: } \\
LOS locus & GBS & MFS & Enteritis & $\boldsymbol{P}$ \\
class & $(\boldsymbol{n}=\mathbf{1 7})$ & $(\boldsymbol{n}=\mathbf{4})$ & $(\boldsymbol{n}=\mathbf{2 1})$ & \\
$\mathrm{A}(n=12)$ & 9 & 0 & 3 & $0.02^{\mathrm{A}}$ \\
$\mathrm{B}(n=14)$ & 3 & 4 & 7 & $0.03^{\mathrm{B}}$ \\
$\mathrm{C}(n=5)$ & 2 & 0 & 3 & NS \\
$\mathrm{D}(n=4)$ & 2 & 0 & 2 & NS \\
$\mathrm{E}(n=7)$ & 1 & 0 & 6 & NS \\
\hline
\end{tabular}

AGBS vs. enteritis. ${ }^{\mathrm{B} M F S}$ vs. enteritis. 


\section{Table 2}

LOS biosynthesis loci and presence of ganglioside-like structures in $C$. jejunistrains

\begin{tabular}{lccccc}
\hline \multicolumn{5}{c}{ No. of strains with a ganglioside-like } \\
structure in the LOS \\
LOS locus & GM1 & GQ1b & 0ther & None & $P$ \\
type & $(\boldsymbol{n}=\mathbf{2 7})$ & $(\boldsymbol{n}=\mathbf{9})$ & $(\boldsymbol{n}=\mathbf{3 0})$ & $(\boldsymbol{n}=\mathbf{8})$ & \\
$\mathrm{A}(n=12)$ & 12 & 1 & 10 & 0 & $<0.01^{\mathrm{B}}$ \\
$\mathrm{B}(n=14)$ & 9 & 8 & 14 & 0 & $<0.01^{\mathrm{C}}$ \\
$\mathrm{C}(n=5)$ & 5 & 0 & 3 & 0 & NS \\
$\mathrm{D}(n=4)$ & 0 & 0 & 1 & 3 & $0.02^{\mathrm{D}}$ \\
$\mathrm{E}(n=7)$ & 1 & 0 & 2 & 5 & $<0.01^{\mathrm{D}}$
\end{tabular}

${ }^{A} A$ strain can express more than 1 ganglioside-like structure in its LOS. Therefore, the sum of strains in a row (GM1 + GQ1b + other + none) is higher than the actual number of strains with the corresponding LOS locus type. BGM1 vs. non-GM1. 'GQ1b vs. non-GQ1b. DNone vs. any.

Sialic acid mutants have a reduced reactivity with patient serum. Analysis of the LOS by gel electrophoresis revealed faster-migrating LOS cores for the GB11 cst-II and orf10/orf11 mutants compared with the WT, confirming that these mutants have a truncated LOS (Figure 4A). The orf11 mutant LOS showed migration patterns identical to those of WT LOS. Results for the GB2 mutant were identical to those obtained with GB11 (data not shown). These findings are concordant with the structures found by mass spectrometry.

To determine whether the gene inactivations influenced the reactivity of the LOS with the antibodies in the serum from GBS patients, we performed an immunoblot analysis with the serum of the GBS patient from whose stools C. jejuni GB11 was isolated (GB11 patient) and WT and mutant purified LOS fractions. Previous studies showed that this serum contains high levels of antiGM1 antibodies (8). Reactivity of GB11 patient serum was reduced for the cst-II and orf10/orf11 mutants (Figure 4B) but remained the same for the orf11 mutant when compared with the WTs. For the cst-II and orf10/orf 11 mutants, the reactivity with cholera toxin, a ligand for GM1-oligosaccharide structures, was almost completely lost (Figure 4C). These results indicate that genes involved in sialic acid biosynthesis and transfer are important for the expression of ganglioside-like LOS structures that are capable of inducing potentially pathogenic autoantibody responses. Although strain GB11 and the "genome" strain NCTC 11168 both express GM1 mimics in their LOS (this was confirmed by their reactivity with cholera toxin; Figure 4C), the GB11 patient serum did not react with the LOS of NCTC 11168 (Figure 4B). This observation has been described previously (17). This observation illustrates the fine specificity of the antibody response in patient GB11, as the GB11 and NCTC 11168 strains have different LOS structures with a common GM1 mimic (Figure 3).

Sialic acid mutants fail to induce anti-ganglioside antibody responses in mice. To demonstrate that the truncated LOS structures in the mutants do not induce autoantibodies, we immunized GD2/ GM2 synthase knockout mice. These mice that lack complex gangliosides are immune-naive hosts that can be used to raise high-titer anti-ganglioside antibody responses (18). Immunization with WT GB11 lyophilized whole bacteria resulted in high IgG serum responses against GD1a, a ganglioside containing 2 sialic acid residues, in 2 of 2 mice (Figure 5A). We could not detect antibody reactivity against GM1 (data not shown). None of the mice injected with the cst-II and orf10/orf11 mutants produced anti-GD1a antibodies (Figure 5, B and C). However, all 6 mice produced anti-asialo-GM1 antibodies, as can be expected from the truncated LOS structures in the cst-II and orf10/orf11 mutants (Figure 5, F and G). Two of 3 mice immunized with the orf11 mutant strain produced GD1a antibodies (Figure 5D). Low asialo-GM1 titers were also found in 3 of 3 mice immunized with the orf 11 mutant (Figure $5 \mathrm{H}$ ). These results indicate that genes involved in sialylation of the LOS are crucial for the induction of cross-reactive anti-ganglioside antibodies.

\section{Discussion}

We have shown that specific types of the C. jejuni LOS biosynthesis gene locus ("LOS locus") are clearly associated with immune-mediated neuropathy and with the presence of ganglioside-mimicking structures in the LOS. Moreover, using Campylobacter gene knockout mutants, we demonstrate here, for the first time to our knowledge, that genes involved in sialylation of the LOS are crucial for the induction of anti-ganglioside antibodies.

Diversity in the content of genes involved in LOS biosynthesis results in the variety of LOS structures that is observed in C. jejuni. In this study, we identified 2 new classes of the LOS locus, in addition to 3 previously described classes $(15,19)$. At the time of writing, DNA sequences of additional C. jejuni strains are accessible through GenBank, and new LOS gene combinations not reported earlier can be identified. However, all our strains had a positive PCR test for 1 of the 5 LOS locus classes described in this study, which indicates that our tests completely covered the spectrum of different LOS loci present in this collection of Campylobacter strains.

The expression of ganglioside-mimicking structures in Campylobacter LOS is considered to be essential for the induction of autoantibodies that lead to GBS. Indeed, we previously reported that ganglioside-mimicking structures are present more frequently in neuropathy-associated strains than in strains isolated from patients with uncomplicated enteritis (8). In contrast, most of the Campylobacter strains whose LOS structure has been chemically characterized and reported in the literature express ganglioside-mimicking structures. The number of strains that have been analyzed, however, is small and may not be representative. Our study demonstrates that C. jejuni needs specific gene combinations to express ganglioside mimics. Only 3 of the 5 identified classes of the LOS locus, i.e., classes A, B, and C, contain genes that are involved in the biosynthesis and transfer of sialic acid, an essential component of gangliosides. We showed that these LOS loci are present in the large majority of strains expressing ganglioside mimics, including the neuropathyassociated strains, whereas class D and E loci are more frequently found in strains lacking ganglioside mimics. In 3 class D/E strains,

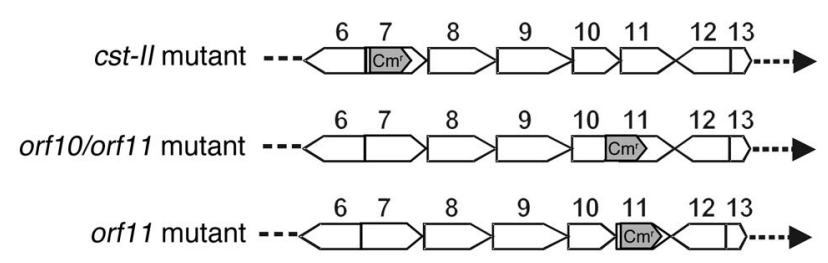

\section{Figure 2}

Schematic representation of the mutants used in this study. Orf6 to orf13 of the class A LOS locus are displayed. The positions of the $0.7-\mathrm{kb}$ $\mathrm{Cm}^{r}$ cassette are indicated with gray arrows. Both orf10 and orf11 are inactivated in the orf10/orf11 mutant. 


\begin{tabular}{|c|c|c|}
\hline Strain & Structure & $\begin{array}{l}\text { Ganglioside } \\
\text { mimic }\end{array}$ \\
\hline \multirow[t]{2}{*}{ WT GB2/GB11 } & $\begin{array}{c}\text { Gal-GalNAc-Gal-Hep-Hep- } \\
\text { I I } \\
\text { NeuAC Glc }\end{array}$ & GM1 \\
\hline & $\begin{array}{l}\text { Gal-GalNAC-Gal-Hep-Hep- } \\
\text { I-Ge I I I } \\
\text { NeuAC NeuAC Glc }\end{array}$ & GD1a \\
\hline \multirow[t]{3}{*}{$\begin{array}{l}\text { cst- } / 1 \text { and orf10/orf } 11 \\
\text { mutants GB2/GB11 }\end{array}$} & $\begin{array}{r}\text { Gal-Hep-Hep- } \\
1 \text { Glc }\end{array}$ & No \\
\hline & $\begin{array}{r}\text { GalNAC-Gal-Hep-Hep- } \\
\text { Glc }\end{array}$ & No \\
\hline & $\begin{array}{r}\text { Gal-GalNAC-Gal-Hep-Hep- } \\
\text { Glc }\end{array}$ & No \\
\hline orf11 mutants GB2/GB11 & like WT & like WT \\
\hline \multirow[t]{2}{*}{ NCTC 11168} & $\begin{array}{c}\text { Gal-GailNAc-Gal-Gal-Hep-Hep- } \\
\text { Ne l } 1 \text { l } 1 \\
\text { NeuAc Gal Glc }\end{array}$ & GM1 \\
\hline & $\begin{array}{c}\text { GalNAC-Gal-Gar-Hep-Hep- } \\
\text { Ne I I I l } \\
\text { NeuAC Gal Glc }\end{array}$ & GM2 \\
\hline
\end{tabular}

ganglioside-like structures other than GM1 or GQ1b were detected (Table 2). The sera used in the serological assay for the detection of these "other" ganglioside-like structures may also react with asialoGM1-like structures lacking sialic acid. Therefore, the detection of an "other" ganglioside-like structure does not necessarily imply that the strain is able to synthesize and transfer sialic acid. However, we cannot exclude the possibility that some strains have both class D or E genes and sialic acid biosynthesis genes that have diverged from their homologues in classes A, B, and C.

Interestingly, we found that the class A locus is associated specifically with GBS and the presence of a GM1-like structure, whereas the class B locus is associated with MFS and the presence of a GQ1b-like structure. The presence of anti-GM1 antibodies has been shown to be associated with a preceding Campylobacter infection (3). We found that GM1-like structures can be expressed by strains with various LOS locus classes. However, the association of a class A LOS locus with both GBS and the presence of a GM1-like structure suggests that class A strains expressing GM1-like LOS structures are more likely to induce GBS compared with non-A strains with a GM1-like LOS.

\section{Figure 4}

SDS-PAGE analysis of LOS from GB11 WT and mutants. The genome strain NCTC 11168 was included as a control. Lane 1, WT GB11; lane 2, GB11 cst-II mutant; lane 3, GB11 orf10/orf11 mutant; lane 4, GB11 orf11 mutant; lane 5, NCTC 11168. (A) Silver staining of the LOS revealed faster-migrating LOS cores for the GB11 cst-I/ and orf10/ orf11 mutants compared with the WT, indicating that these mutants have a truncated LOS. The orf11 mutant LOS showed migration patterns identical to those of WT LOS. (B) A Western blot incubated with GB11 patient serum showed a reduced reactivity for the cst-// and orf10/orf11 mutants but unchanged reactivity for the orf11 mutant when compared with the WTs. (C) For the cst-II and orf10/orf11 mutants, the reactivity with cholera toxin, a ligand for GM1-oligosaccharide structures, was almost completely lost. Reactivity with the orf11 mutant remained unchanged.

\section{Figure 3}

Molecular mimicry between gangliosides and LOS outer cores of WT and mutant $C$. jejuni strains. LOS outer core structures of GB2 and GB11 WT and mutant strains were determined by mass spectrometry analysis. The LOS outer core structure of the "genome" strain NCTC 11168 was described by St. Michael et al. (35). Note that all strains express a mixture of different LOS structures. The cst-Il and orf10/ orf11 mutants of GB2 and GB11 expressed not ganglioside mimics but a mixture of 3 nonsialylated structures. No structural differences were observed between the WTs and the orf11 mutants.

MFS, a variant of GBS, has consistently been associated with preceding C. jejuni infection. Several authors have demonstrated that GQ1b-like structures occur more frequently in MFS-associated strains and are associated with anti-GQ1b antibody reactivity and the presence of oculomotor symptoms in patients with GBS or MFS $(8,20)$. Although GQ1b-like structures were detected in both class A and class B strains, we found that only the class B locus was significantly associated with the presence of a GQ1b-like structure and that all our MFS-associated strains contained a class B locus. However, not all class B strains expressed GQ1b-like structures. This may be explained by mechanisms such as phase variation or point mutations that lead to gene inactivations resulting in the absence of expression of GQ1b-like structures in some class B strains $(21,22)$. In conclusion, we have now identified specific molecular substrates in C. jejuni that are involved in the induction of GBS or MFS.

To our knowledge, this is the first study that analyzed the LOS locus of a large collection of neuropathy-associated and control C. jejuni strains. Previously, it was demonstrated that 2 closely related GBS-associated strains contained a class A locus (16). Very recently, we demonstrated that the strain GB11 that we used in this study has probably acquired its class A locus through horizontal gene transfer (17). Another recent report did not find an association between GBS and LOS biosynthesis genes using a whole-genome DNA microarray (23). This microarray, however, only contained LOS genes present in a class $\mathrm{C}$ locus and would therefore not be able to detect the association described here.

Based on the results of the LOS locus analysis, we hypothesized that genes unique to the class $\mathrm{A}$ and $\mathrm{B}$ loci or genes that are involved in sialic acid biosynthesis and transfer are crucial for the induction of neuropathogenic cross-reactive antibodies. To test this hypoth-

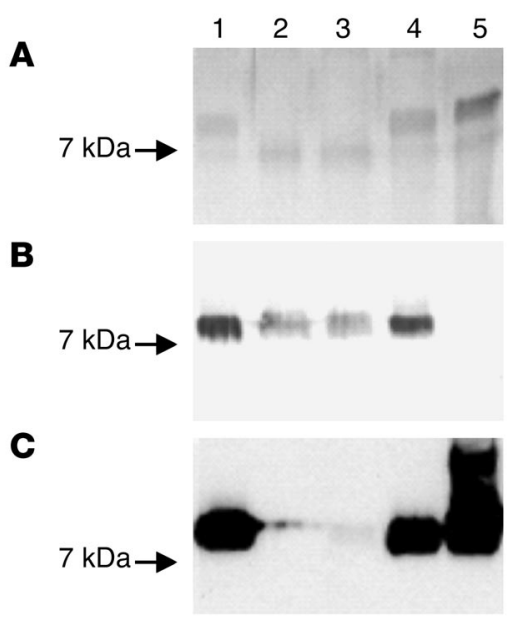




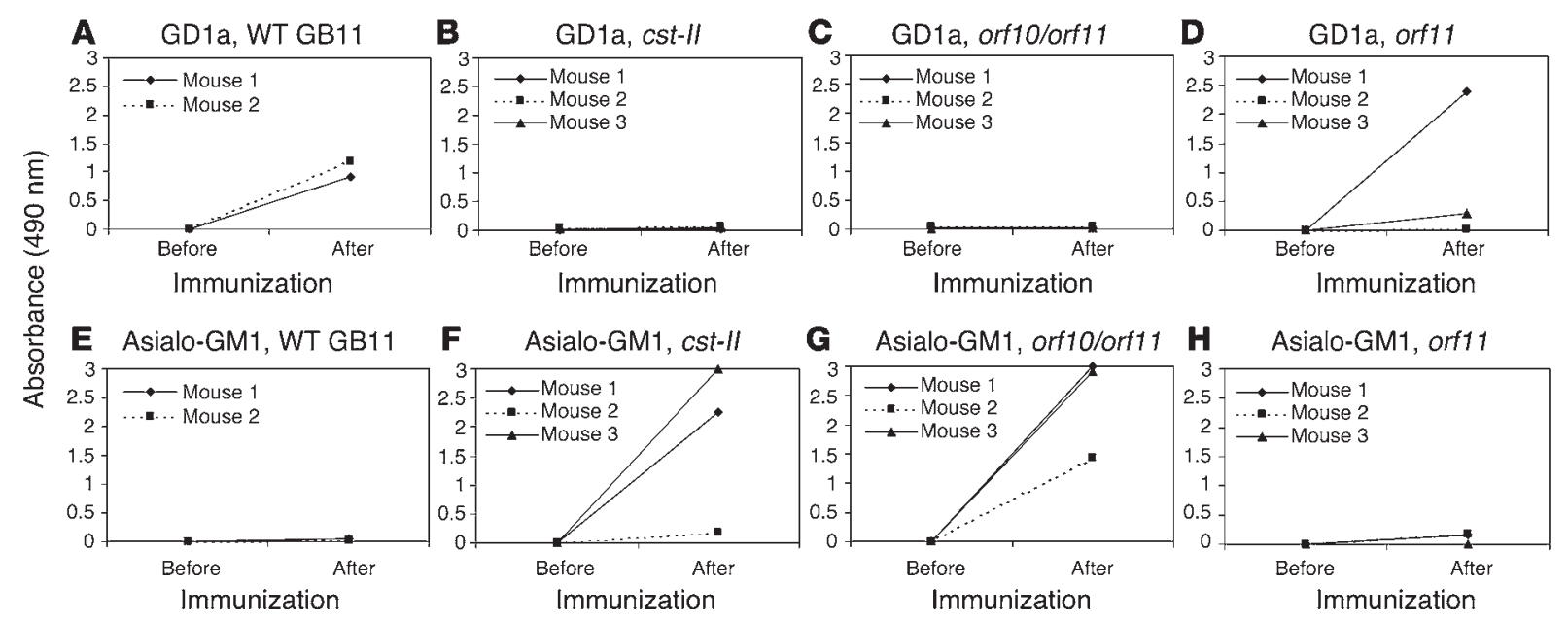

Figure 5

Anti-glycolipid antibodies in serum of mice before and after immunization with WT and mutant GB11 C. jejuni strains. (A and D) When sialic acid was present on the LOS core (WT and orf11 mutant), anti-GD1a antibodies were induced in mice. (B and C) Anti-GD1a antibody responses were not detected in mice immunized with nonsialylated LOS (cst-II and orf10/orf11 mutants). (F and $\mathbf{G})$ Nonsialylated LOS (cst-II and orf10/orf11 mutants) induced high asialo-GM1 antibody responses. (E and $\mathbf{H}$ ) Asialo-GM1 antibody responses were absent or low after immunization with the orf11 mutant and WT.

esis, we constructed a Campylobacter knockout mutant of orf11, encoding a putative acetyltransferase with unknown function in LOS biosynthesis and the only gene that is unique to class $\mathrm{A}$ and $\mathrm{B}$ loci. Several genes are involved in the sialylation of Campylobacter LOS (Supplemental Table 1). Inactivation of either of these genes will probably lead to identical changes in LOS structure; i.e., truncated LOS lacking sialic acid. We constructed knockout mutants of 2 such genes: orf10, encoding a CMP-sialic acid synthetase, and cst-II. Cst-II, present in class A and B loci, encodes a bifunctional sialic acid transferase that has been characterized in detail and that has been associated with the presence of a GQ1b-like structure $(13,15,24)$. Cst-II has a sialyltransferase homologue in the class C locus, called cst-III (53\% identity), but the bifunctional ( $\alpha-2,3 /$ $\alpha-2,8)$ sialyltransferase activity that is probably necessary for the biosynthesis of GQ1b-like structures has so far only been detected for cst-II (15). Orf10 was inactivated along with the adjoining orf11 in a so-called double mutant (Figure 2). Because the orf11 single mutants did not show any differences compared with the WTs, we assumed that the differences observed in the orf10/orf11 double mutant were the result of the inactivation of orf10. Mutants were constructed of 2 class A GBS-associated strains that expressed GM1-like structures in their LOS. We were unsuccessful in our efforts to construct mutants of MFS-associated strains expressing GQ1b-like structures, since, unfortunately, these strains did not appear to be transformable (data not shown).

Mass spectrometry analysis revealed a mixture of 2 different sialylated ganglioside-mimicking structures in the WT GB2 and GB11 strains. Inactivation of cst-II and orf10 resulted in a mixture of 3 truncated LOS structures lacking sialic acid. Two of these structures lacked not only sialic acid, when compared with the WT, but also the outer Gal or Gal-GalNAc residues of the LOS backbone (Figure 3). This observation is consistent with the lower activity of the $\beta-1,4-N$-acetylgalactosaminyltransferase (CgtA) on nonsialylated acceptors that was previously reported for CgtA from C. jejuni OH4384 (16). Inactivation of orf11 did not result in differences in LOS structure compared with that in the WT strains. Therefore, the function, if any, of this gene in LOS biosynthesis and in the pathogenesis of GBS remains unknown. In summary, mass spectrometry analysis revealed that genes involved in sialylation are necessary for the biosynthesis of ganglioside mimics in Campylobacter LOS. Other studies have demonstrated the role of cst-III (orf7c), neuB1 (orf8c), and neuC1 (orf9b and orf9c) in the synthesis of ganglioside mimics $(21,25,26)$.

Inactivation of cst-II and orf10 resulted in reduced reactivity of GB11 patient serum with mutant LOS, providing support for their importance in the induction of autoantibodies in GBS. Although residual reactivity was seen, this was probably caused by anti-asialo-GM1 antibodies or anti-GM1 and antiGD1b antibodies that cross-react with asialo-GM1 structures in the mutant LOS. It has previously been demonstrated that the GB11 patient serum contains antibodies directed against GM1 (high titer), asialo-GM1 (low titer), and GD1b (high titer), which all contain the terminal disaccharide Gal-GalNAc (8). Although nonsialylated glycoconjugates are present on nerve tissue, so far, anti-asialo-GM1 antibodies have not been associated with GBS. The complete absence of reactivity of cholera toxin with cst-II and orf10/orf11 mutant LOS further confirms the crucial role of cst-II and orf10 in the biosynthesis of ganglioside mimics.

The mouse immunization experiments conducted in this study provide additional evidence for the necessity of sialylation genes for the induction of autoantibodies. Both cst-II and orf10/orf11 mutants failed to induce anti-ganglioside antibodies in mice, in contrast to the WT strains. Mice injected with the cst-II and orf10/ orf11 mutants produced serum antibodies against the nonsialylated glycoconjugate asialo-GM1, but these antibodies have not yet been associated with GBS. Anti-GD1a but no anti-GM1 antibodies were detected in mice immunized with the GB11 WT strain. This is in contrast with earlier findings that GB11 also expresses a GM1-like LOS structure and that GB11 patient serum contains high levels of anti-GM1 antibodies. However, it has been observed previously that immunization with both GD1a and GM1 induced primarily anti-GD1a responses in mice, whereas rabbits only had 
anti-GM1 antibodies $(27,28)$. Thus, anti-ganglioside antibody responses may be species dependent.

The associations that we found are not absolute. As host factors also play an important role in the pathogenesis of GBS, infection with a C. jejuni strain that expresses ganglioside-like structures is not sufficient to trigger GBS. In addition, a variety of ganglioside mimics, produced by different LOS classes, have been associated with GBS with diverse clinical manifestations $(8,20,29)$. Furthermore, it is not clear at present whether all bacterial factors involved in the development of GBS/MFS have been identified.

After the demonstration of anti-ganglioside antibodies in GBS/ MFS patient sera, and the subsequent identification of ganglioside mimics in Campylobacter LOS and of the specific host responses toward these epitopes, we now also demonstrate that the microbial gene repertoire is an important factor in the initiation of postinfectious autoimmune disease. The markers described in the current communication may facilitate the search for mechanisms of microbial pathogenicity and be helpful in the development of new molecular diagnostic tools for identifying C. jejuni strains with an increased ability to induce GBS/MFS. Furthermore, increased insight into the biosynthesis of ganglioside-mimicking structures may ultimately lead to the development of new treatment strategies and interventions (30).

\section{Methods}

C. jejuni strains. Seventeen GBS-associated and 4 MFS-associated C. jejuni strains used in this study were isolated from GBS/MFS patients from The Netherlands and Belgium between 1991 and 1999 (10). GB13 and GB26 were cultured from the diarrheal stools of family members of 2 GBS patients (31). Twenty-one control C. jejuni strains were isolated from sporadic Dutch enteritis patients without neurological symptoms between 1990 and 1999 (8). Ten reference C. jejuni Penner serotypes (HS:1, HS:2, HS:3, HS:4, HS:10, HS:19, HS:23, HS:35, HS:36, and HS:64) and the "genome" strain NCTC 11168 (19) were also included as controls for the PCR tests. Characterization of the isolates by phenotypic and molecular methods showed that no clustering of GBS/MFS-associated strains occurred when these were compared with control strains $(10,12)$.

Growth conditions and DNA isolation. For PCR analysis, C. jejuni strains were cultured for 24-48 hours on blood agar plates in a microaerobic atmosphere at $37^{\circ} \mathrm{C}$. DNA was isolated using the Wizard Genomic DNA Purification Kit (Promega Corp.).

For other experiments, C. jejuni WT strains were grown at $37^{\circ} \mathrm{C}$ on Mueller-Hinton agar plates supplemented with Modified Preston Campylobacter Selective Supplement (MPCSS; product code SR0204E; Oxoid Ltd.) in a microaerobic atmosphere. The mutant strains were grown on Mueller-Hinton agar plates supplemented with MPCSS and chloramphenicol, at a concentration of $20 \mu \mathrm{g} / \mathrm{ml}$. For cloning, the E. coli strain JM109 (Promega Corp.) was grown on 2YT agar with $40 \mu \mathrm{g} / \mathrm{ml}$ ampicillin and $20 \mu \mathrm{g} / \mathrm{ml}$ chloramphenicol.

Serological methods. The ganglioside mimics in the LOS of the various strains were determined using polyclonal and monoclonal anti-ganglioside antibodies as described previously (8). An inventory was made for GM1like, GQ1b-like, and, collectively, other ganglioside-like structures.

Determination of the LOS locus class. To determine the class of LOS locus in C. jejuni strains, we developed specific primer sets for the classes A/B, $\mathrm{C}, \mathrm{D}$, and $\mathrm{E}$, based on the DNA sequence of a gene unique to the LOS locus class(es) involved (Supplemental Table 1). To discern between classes A and B, we developed a primer set that was based on the DNA sequence of orf5-II. PCR assays were performed using a Thermocycler 60 (Biomed $\mathrm{GmbH}$ ) with a program consisting of 40 cycles of 1 minute at $94^{\circ} \mathrm{C}, 1$ minute at $52^{\circ} \mathrm{C}$, and 2 minutes at $74^{\circ} \mathrm{C}$. Per reaction, approximately $50 \mathrm{ng}$ of template DNA was used in a buffer system consisting of $10 \mathrm{mM}$ Tris- $\mathrm{HCl}$ ( $\mathrm{pH} 9.0$ ), $50 \mathrm{mM} \mathrm{KCl}, 1.5 \mathrm{mM} \mathrm{MgCl}_{2}, 0.01 \%$ gelatin, $0.1 \%$ Triton X-100, $0.2 \mathrm{mM}$ of each of the deoxyribonucleotide triphosphates (Promega Corp.), and 0.2 U Super Taq polymerase (HT Biotechnology Ltd.). Timing needed to be adjusted for some of the amplifications. The selective PCR tests were validated with a panel of C. jejuni strains with a known LOS locus class.

Cloning and mutagenesis. The target genes (cst-II, orf10/orf11, and orf11) and approximately $700 \mathrm{bp}$ of flanking sequence were amplified and cloned into the pGem-Teasy vector (Promega Corp.). Inverse PCR was used to introduce a $\mathrm{BamHI}$ restriction site and a deletion of approximately $800 \mathrm{bp}$ in the target genes (32). Inverse PCR products were digested with $B a m H I$ and ligated to the BamHI-digested chloramphenicol resistance $\left(\mathrm{Cm}^{\mathrm{r}}\right)$ cassette of pAV35 (33). After sequencing, the resulting constructs were electroporated into different $C$. jejuni strains, and recombinants were selected on Mueller-Hinton plates containing chloramphenicol (33). Junction PCR, with primers up- and downstream of the area involved in the homologous recombination, and primers in the $\mathrm{Cm}^{\mathrm{r}}$ cassette, was performed to confirm double crossover events and to assess the orientation of the resistance cassette.

Mass spectrometric analysis. Overnight growths from 1 agar plate were treated as described by Szymanski et al. (34) except that we used proteinase $\mathrm{K}$ at $60 \mu \mathrm{g} / \mathrm{ml}$, RNase A at $200 \mu \mathrm{g} / \mathrm{ml}$, and DNase I at 100 $\mu \mathrm{g} / \mathrm{ml}$. The $O$-deacylated LOS samples were analyzed by capillary electrophoresis coupled with electrospray ionization mass spectrometry (CE-ESI-MS) as described by St. Michael et al. (35).

SDS-PAGE and Western blotting. Fresh overnight cultures of GB11 WT and mutant strains were harvested in PBS and lysed by sonification. To visualize both protein and LOS fractions, the cell lysates were electrophoresed on $11 \%$ SDS-PAGE gels. To visualize the LOS fraction only, cell lysates were treated with proteinase $\mathrm{K}(1 \mathrm{mg} / \mathrm{ml})$ for 2 hours at $65^{\circ} \mathrm{C}$ and electrophoresed on $15 \%$ SDS-PAGE gels. Gels were silver-stained to visualize LOS cores, or the gels were blotted onto nitrocellulose. GB11 patient serum was used at a dilution of 1:2,500, and peroxidase-conjugated cholera toxin (Sigma-Aldrich) was used at a concentration of $1 \mu \mathrm{g} / \mathrm{ml}$.

Mouse immunization experiments. This research was approved by the Animal Care and Use Committee, Dokkyo University School of Medicine (approval no. 00-22). Mice were treated according to the Guidelines for the Care and Use of Laboratory Animals of Dokkyo University School of Medicine. Mice lacking GM2/GD2 synthase as well as GM1 and GD1a (36) were immunized i.p. several times at 2-week intervals with $1 \mathrm{mg}$ of each lyophilized C. jejuni dissolved in $100 \mu \mathrm{l}$ keyhole limpet hemocyanin solution, mixed with $100 \mu \mathrm{lCFA}$. One week after the final inoculation, blood samples were taken from the tail vein. Serum IgG antibodies to GM1, GD1a, and asialoGM1 were measured by an ELISA (37). The mean absorbance value for triplicate reference wells without antigen was subtracted from the mean value for triplicate sample wells with the antigen.

Statistical analysis. Statistical analysis was performed with InStat (version 2.05a; GraphPad Software Inc.) using $2 \times 2$ contingency tables. Fisher's exact tests were executed and 2 -sided $P$ values determined. Associations were considered significant at $P<0.05$.

\section{Acknowledgments}

We thank Arnoud van Vliet for technical advice and Wouter van Rijs and Frank St. Michael for technical assistance. We are grateful to the microbiologists and neurologists participating in the Dutch Guillain-Barré trial for their efficient logistic support in providing fecal specimens. This study was supported by grants from The Netherlands Organization for Scientific Research 
(920-03-225, to P.C.R. Godschalk) and from the Human Frontier Science Program (RGP 38/2003).

Received for publication April 16, 2002, and accepted in revised form September 28, 2004.

Address correspondence to: Peggy C.R. Godschalk, Department of Medical Microbiology and Infectious Diseases, Erasmus MC, Dr. Molewaterplein 40, 3015 GD Rotterdam, The Neth- erlands. Phone: 31-10-4633510; Fax: 31-10-4633875; E-mail: p.godschalk@erasmusmc.nl.

Peggy C.R. Godschalk and Astrid P. Heikema contributed equally to this work.

This work was presented in part at the 12th International Workshop on Campylobacter, Helicobacter and Related Organisms in Aarhus, Denmark, on September 6-10, 2003.
1. Albert, L.J., and Inman, R.D. 1999. Molecular mimicry and autoimmunity. N. Engl. J. Med. 341:2068-2074.

2. Ang, C.W., Jacobs, B.C., and Laman, J.D. 2004. The Guillain-Barré syndrome: a true case of molecular mimicry. Trends Immunol. 25:61-66.

3. Yuki, N. 2001. Infectious origins of, and molecular mimicry in, Guillain-Barré and Fisher syndromes. Lancet Infect. Dis. 1:29-37.

4. Jacobs, B.C., et al. 1998. The spectrum of antecedent infections in Guillain-Barré syndrome: a casecontrol study. Neurology. 51:1110-1115.

5. Lecuit, M., et al. 2004. Immunoproliferative small intestinal disease associated with Campylobacter jejuni. N. Engl. J. Med. 350:239-248.

6. Willison, H.J., and Yuki, N. 2002. Peripheral neuropathies and anti-glycolipid antibodies. Brain. 125:2591-2625.

7. Moran, A.P. 1997. Structure and conserved characteristics of Campylobacter jejuni lipopolysaccharides. J. Infect. Dis. 176(Suppl. 2):S115-S121.

8. Ang, C.W., et al. 2002. Structure of Campylobacter jejuni lipopolysaccharides determines antiganglioside specificity and clinical features of GuillainBarré and Miller Fisher patients. Infect. Immun. 70:1202-1208.

9. Moran, A.P., Rietschel, E.T., Kosunen, T.U., and Zahringer, U. 1991. Chemical characterization of Campylobacter jejuni lipopolysaccharides containing $\mathrm{N}$-acetylneuraminic acid and 2,3-diamino-2,3dideoxy-D-glucose. J. Bacteriol. 173:618-626.

10. Endtz, H.P., et al. 2000. Molecular characterization of Campylobacter jejuni from patients with GuillainBarré and Miller Fisher syndromes. J. Clin. Microbiol. 38:2297-2301.

11. Fisher, M. 1956. An unusual variant of acute idiopathic polyneuritis: syndrome of ophthalmoplegia, ataxia and areflexia. N. Engl. J. Med. 255:57-65.

12. Dingle, K.E., et al. 2001. Sequence typing confirms that Campylobacter jejuni strains associated with Guillain-Barré and Miller Fisher syndromes are of diverse genetic lineage, serotype and flagella type. J. Clin. Microbiol. 39:3346-3349.

13. van Belkum, A., et al. 2001. A Campylobacter jejuni gene associated with immune-mediated neuropathy. Nat. Med. 7:752-753.

14. Linton, D., Karlyshev, A.V., and Wren, B.W. 2001. Deciphering Campylobacter jejuni cell surface interactions from the genome sequence. Curr. Opin. Microbiol. 4:35-40.

15. Gilbert, M., et al. 2002. The genetic bases for the variation in the lipo-oligosaccharide of the mucosal pathogen, Campylobacter jejuni. Biosynthesis of sialylated ganglioside mimics in the core oligosaccharide. J. Biol. Chem. 277:327-337.

16. Gilbert, M., et al. 2000. Biosynthesis of ganglioside mimics in Campylobacter jejuni OH4384. Identification of the glycosyltransferase genes, enzymatic synthesis of model compounds, and characterization of nanomole amounts by $600-\mathrm{MHz}^{1} \mathrm{H}$ and ${ }^{13} \mathrm{C}$ NMR analysis. J. Biol. Chem. 275:3896-3906.

17. Gilbert, M., et al. 2004. Evidence for acquisition of the lipooligosaccharide biosynthesis locus in Campylobacter jejuni GB11, a strain isolated from a patient with Guillain-Barré syndrome, by horizontal exchange. Infect. Immun. 72:1162-1165.

18. Lunn, M.P.T., et al. 2000. High-affinity antiganglioside IgG antibodies raised in complex ganglioside knockout mice. Reexamination of GD1a immunolocalization. J. Neurochem. 75:404-412.

19. Parkhill, J., et al. 2000. The genome sequence of the food-borne pathogen Campylobacter jejuni reveals hypervariable sequences. Nature. 403:665-668.

20. Chiba, A., Kusunoki, S., Obata, H., Machinami, R., and Kanazawa, I. 1993. Serum anti-GQ1b IgG antibody is associated with ophthalmoplegia in Miller Fisher syndrome and Guillain-Barré syndrome: clinical and immunohistochemical studies. Neurology. 43:1911-1917.

21. Guerry, P., et al. 2002. Phase variation of Campylobacter jejuni 81-176 lipooligosaccharide affects ganglioside mimicry and invasiveness in vitro. Infect. Immun. 70:787-793.

22. Prendergast, M.M., et al. 2004. In vivo phase variation and serologic response to lipooligosaccharide of Campylobacter jejuni in experimental human infection. Infect. Immun. 72:916-922.

23. Leonard, E.E., II, Tompkins, L.S., Falkow, S., and Nachamkin, I. 2004. Comparison of Campylobacter jejuni isolates implicated in Guillain-Barré syndrome and strains that cause enteritis by a DNA microarray. Infect. Immun. 72:1199-1203.

24. Chiu, C.P., et al. 2004. Structural analysis of the sialyltransferase CstII from Campylobacter jejuni in complex with a substrate analog. Nat. Struct. Mol. Biol. 11:163-170.

25. Guerry, P., Ewing, C.P., Hickey, T.E., Prendergast, M.M., and Moran, A.P. 2000. Sialylation of lipooligosaccharide cores affects immunogenicity and serum resistance of Campylobacter jejuni. Infect. Immun. 68:6656-6662.
26. Linton, D., et al. 2000. Multiple N-acetyl neuraminic acid synthetase (neuB) genes in Campylobacter jejuni: identification and characterization of the gene involved in sialylation of lipo-oligosaccharide. Mol. Microbiol. 35:1120-1134.

27. Bowes, T., et al. 2002. Tolerance to self gangliosides is the major factor restricting the antibody response to lipopolysaccharide core oligosaccharides in Campylobacter jejuni strains associated with GuillainBarré syndrome. Infect. Immun. 70:5008-5018.

28. Yuki, N., et al. 2001. Animal model of axonal Guillain-Barré syndrome induced by sensitization with GM1 ganglioside. Ann. Neurol. 49:712-720.

29. Jacobs, B.C., et al. 1996. Campylobacter jejuni infections and anti-GM1 antibodies in Guillain-Barré syndrome. Ann. Neurol. 40:181-187.

30. Willison, H.J., et al. 2004. Synthetic disialylgalactose immunoadsorbents deplete anti-GQ1b antibodies from autoimmune neuropathy sera. Brain. 127:680-691

31. Ang, C.W., et al. 2000. A case of Guillain-Barré syndrome following a family outbreak of Campylobacter jejuni enteritis. J. Neuroimmunol. 111:229-233.

32. Wren, B.W., Henderson, J., and Ketley, J.M. 1994. A PCR-based strategy for the rapid construction of defined bacterial deletion mutants. Biotechniques. 16:994-996.

33. van Vliet, A.H., Wooldridge, K.G., and Ketley, J.M. 1998. Iron-responsive gene regulation in a Campylobacter jejuni fur mutant. J. Bacteriol. 180:5291-5298.

34. Szymanski, C.M., et al. 2003. Detection of conserved $\mathrm{N}$-linked glycans and phase-variable lipooligosaccharides and capsules from Campylobacter cells by mass spectrometry and high resolution magic angle spinning NMR spectroscopy. J. Biol. Chem. 278:24509-24520.

35. St. Michael, F., et al. 2002. The structures of the lipooligosaccharide and capsule polysaccharide of Campylobacter jejuni genome sequenced strain NCTC 11168. Eur. J. Biochem. 269:5119-5136.

36. Takamiya, K., et al. 1996. Mice with disrupted GM2/GD2 synthase gene lack complex gangliosides but exhibit only subtle defects in their nervous system. Proc. Natl. Acad. Sci. U. S. A. 93:10662-10667.

37. Yuki, N., Tagawa, Y., Irie, F., Hirabayashi, Y., and Handa, S. 1997. Close association of Guillain-Barré syndrome with antibodies to minor monosialogangliosides GM1b and GM1 alpha. J. Neuroimmunol. 74:30-34. 